Review article

\title{
MOLTO TARDI MA TROPPO PRESTO... IL PROF. ANGELO CHIAVARO E LA LAUREA IN ODONTOIATRIA ITALIANA
}

\author{
VERY LATE BUT TOO EARLY... \\ PROF. ANGELO CHIAVARO AND THE \\ ITALIAN DEGREE IN DENTISTRY
}

\author{
Stefano Eramo*, Caterina Bensi", Stefano Belli", Stefano Pagano**
}

\begin{abstract}
SUMMARY
The birth of the Degree in Dentistry in Italy has been very troubled, and only in 1980 saw its effective implementation. Very "instructive" in this regard is the history on the establishment in 1924 (the period of the seizure of power by Fascism) of a "National School of Dentistry" at the University of Rome, which was withdrawn after only ten months. The biggest supporter and proponent of the School, Prof. Angelo Chiavaro, after a few years, was "punished" with the transfer from the University of Rome to that of Genoa. We present some brief notes on the biography of this courageous pioneer and the matter of which he was the protagonist.
\end{abstract}

Keywords: Chiavaro; dentistry; dental degree.

Università degli Studi di Perugia, Dipartimento di Scienze Chirurgiche e Biomediche, Corso di Laurea Magistrale in Odontoiatria e Protesi dentaria - Insegnamento di Propedeutica Clinica Odontostomatologica.

** Università degli Studi di Perugia, Dipartimento di Scienze Chirurgiche e Biomediche, Corso di Laurea Magistrale in Odontoiatria e Protesi dentaria - Insegnamento di Materiali dentari.

Corrispondenza: Stefano Eramo, Università degli Studi di Perugia, Dipartimento di Scienze Chirurgiche e Biomediche, Piazza Lucio Severi 1 - 06132 Perugia, Italia. E-mail: stefano.eramo@alice.it. 


\section{INTRODUZIONE}

Il I8 gennaio 1924, con la pubblicazione sulla Gazzetta Ufficiale del Regno d'Italia, diventava esecutivo il Regio Decreto del 3I dicembre 1923, $\mathrm{n}^{\circ} 2910$, riguardante l'"Istituzione di una Regia scuola Nazionale di Odontoiatria annessa alla Regia Università di Roma”. Il Decreto portava le firme del re Vittorio Emanuele III, del Capo del Governo Mussolini e dei Ministri Gentile e De' Stefani (fig.r). L'istituzione della Scuola, che era destinata a

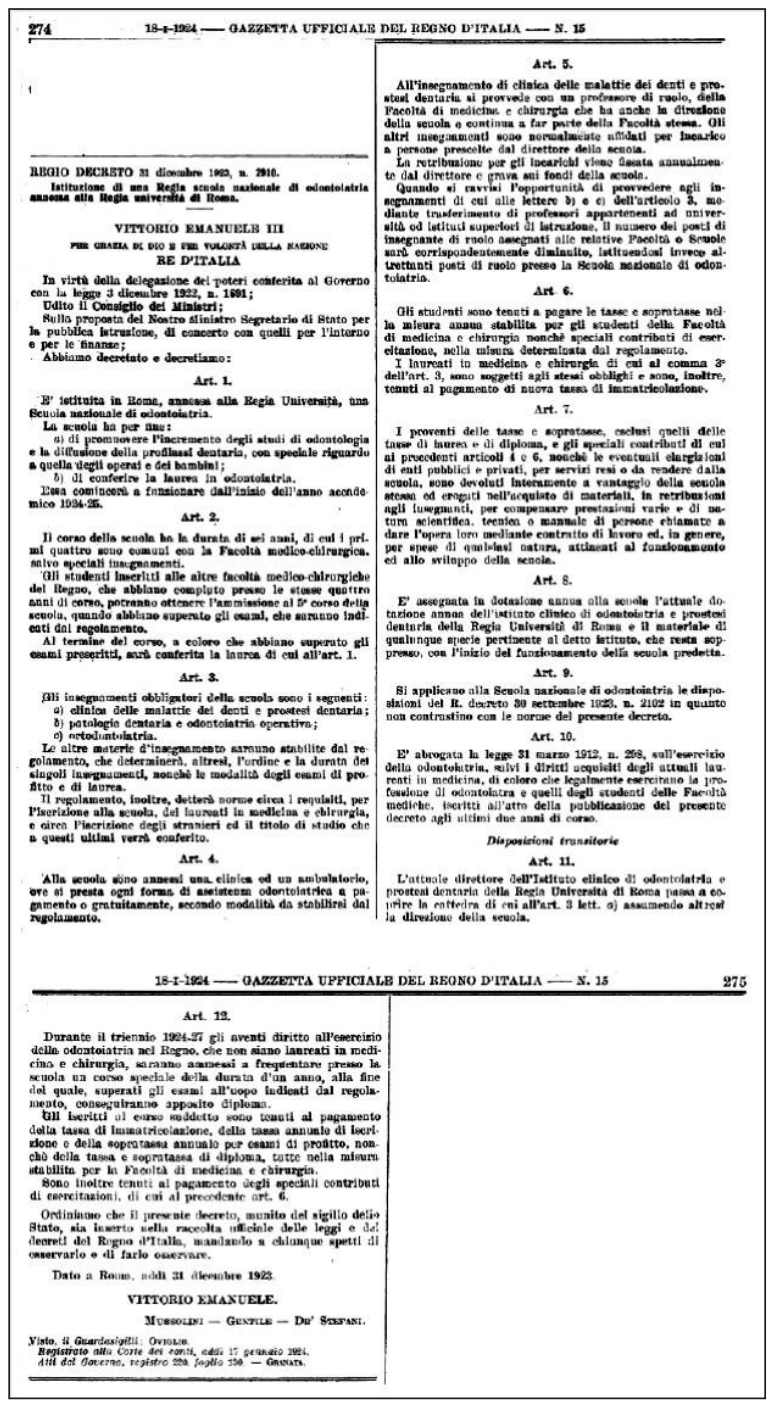

Fig. I - Copia originale del Regio Decreto del 3r dicembre 1923, n² $2910-$ Regno d'Italia. 
rilasciare la Laurea in Odontoiatria che sino ad allora non esisteva in Italia, seguiva dopo molti anni quanto era avvenuto negli Stati Uniti d'America e in vari paesi europei: la Prima Scuola di Odontoiatria autonoma venne infatti fondata nel 1840 a Baltimora (I), seguita da numerose altre nel trentennio I845-1877, sempre negli Stati Uniti (2); a Parigi, nel r88o, fu fondata l'"Ecole Dentaire" (3); a Berlino, nel r884 lo "Zahnaertzliche Poliklinik" (4); a Londra, nel 1887 la "Guy's Hospital Dental School" (5) ecc. Il Decreto nasceva su impulso del Ministro per la Pubblica Istruzione prof. Giovanni Gentile, uno dei maggiori filosofi che l'Italia abbia mai avuto, ma era dovuto al lavoro infaticabile dell'allora Direttore dell'Istituto Clinico di Odontoiatria e protesi dentaria della Regia Università di Roma, il prof. Angelo Chiavaro. Sulla figura di Chiavaro, quasi dimenticata in Italia salvo qualche lodevole eccezione (6-8), abbiamo voluto incentrare questo lavoro soprattutto perché il suo adoperarsi per un'Odontoiatria sorella ma indipendente dalla Medicina gli costò molto, come vedremo, in termini personali.

\section{Cenni biografici}

Angelo Chiavaro (fig. 2) nacque a Catania nel r870, si laureò in Medicina e Chirurgia a Firenze nel 1895, discutendo la tesi "Congiuntivite blenorragica ed acquisita dei neonati"; dopo la laurea, sempre a Firenze, frequentò i corsi di perfezionamento in Patologia generale e in Clinica chirurgica, e nel 1896 vinse il posto di "Medico Chirurgo astante volontario per le malattie di bocca e denti" nell'arcispedale di S. Maria Nuova (9). Questo lo portò a dedicarsi in maniera approfondita allo studio dell'Odontoiatria, e il suo interesse per la disciplina aumentò rapidamente tanto da indurlo, nel 1897 , anche su indicazione di un dentista americano famoso a Firenze, il dott. William Dunn,

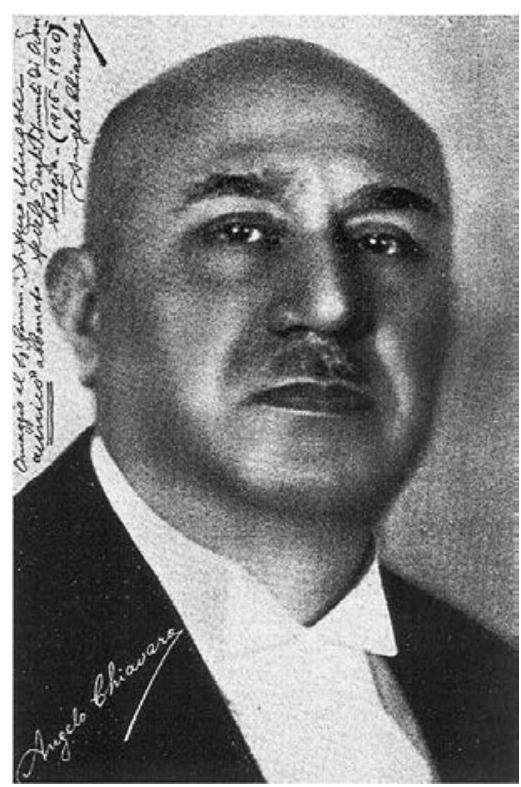

Fig. 2 - Ritratto fotografico del prof. Angelo Chiavaro. a recarsi negli Stati Uniti (9); qui fu il primo medico italiano a iscriversi e a laurearsi alla Scuola Dentaria di Filadelfia, ottenendo nel I899 il diploma di 
dottore in chirurgia dentaria (Io). Dopo aver superato gli esami di Stato per l'abilitazione all'esercizio della Chirurgia dentaria e della Medicina e Chirurgia nello Stato di Pennsylvania, fino al rgor esercitò la professione a Filadelfia; in questo periodo inventò una nuova leva dentaria, con giunto a sfera cri-

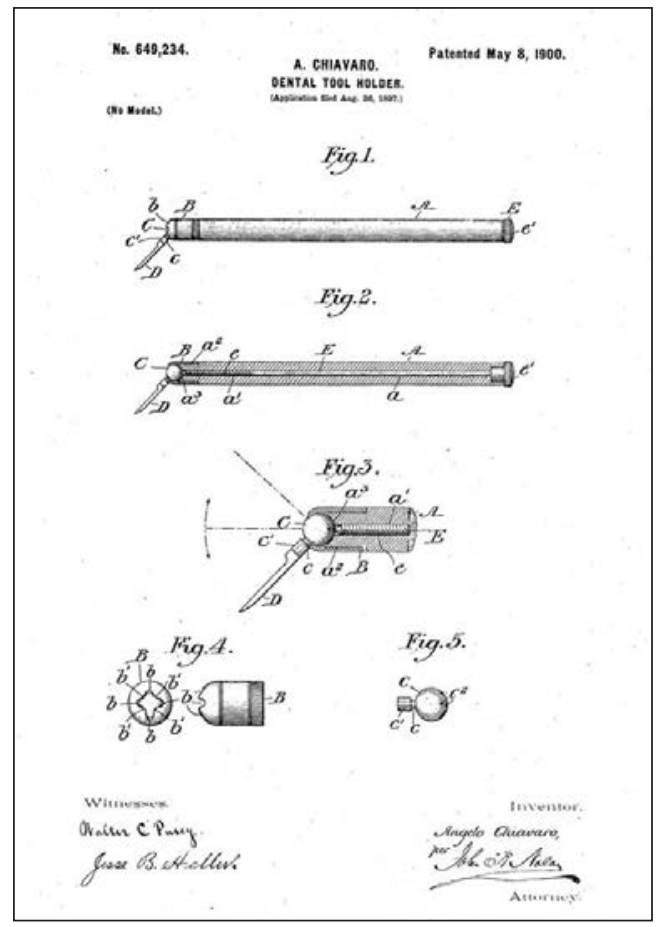

Fig. 3 - Copia del brevetto di una nuova leva dentaria, con giunto a sfera cribrosa di A. Chiavaro. brosa rigidamente flessibile (fig. 3). Chiavaro tornò in Italia nel 1903, dopo un biennio trascorso a visitare le varie scuole odontoiatriche europee (Spagna, Francia, Belgio, Olanda, Inghilterra, Finlandia, Russia, Germania -ove seguì le lezioni di W.D. Miller, Svizzera e AustriaUngheria) e si stabilì a Roma aprendo uno studio privato che presto fu tra i più frequentati della capitale italiana, fermo nell'intenzione di dedicarsi all'insegnamento dell'Odontoiatria che, nell'Italia del periodo, era - nella maggior parte dei casi -in mano a cavadenti e frati (II). $\mathrm{Nel}{ }_{904} \mathrm{fu}$ inserito nella Commissione ministeriale per lo studio dell'organizzazione degli studi e della pratica professionale Odontoiatrici in Italia. Nel 1905 conseguì a Roma la libera docenza in Odontoiatria e Protesi dentaria e nel 1908 ottenne l'incarico dell'insegnamento di Odontoiatria e Protesi dentaria nell'Università di Roma, che gli fu poi rinnovato fino al Igr4 (7). Nel I9I5 vinse il concorso come Professore Ordinario per la prima cattedra di Odontoiatria e Protesi dentaria del Regno d'Italia, istituita presso l'Università di Roma, e ne mantenne la direzione fino al I928, quando venne sottoposto a trasferimento di ufficio all'Università di Genova, dove continuò ad insegnare fino al 1940, anno del suo collocamento a riposo per raggiunti limiti di età. Angelo Chiavaro morì ad Acqui il 25 luglio 1944 (Io). In cinquant'anni di attività didattica e scientifica Chiavaro pubblicò oltre duecento articoli di argomento odontoiatrico e numerosi 
altri in cui si occupò della riforma degli studi universitari. Nel I916 fondò il periodico "Annali di odontologia", che diresse fino al 1940 (ma che dopo il I928 prese il nome di "Archivio Chiavaro") ed i cui scopi dichiarati erano quelli di dare maggiore impulso alla letteratura odontoiatrica, di diffondere le nuove cognizioni e applicazioni pratiche, di far conoscere ed apprezzare all'estero l'attività scientifica degli odontoiatri italiani. Fu autore del capitolo "Semeiotica e diagnostica speciale odontoiatrica" nel Trattato di semeiologia fisica e diagnostica chirurgica generale e speciale, diretto da D. Taddei (I2). Nei numerosi lavori scientifici il C. si occupò di quasi tutti gli aspetti dell'Odontoiatria - embriologia, istologia, fisiologia, parassitologia, patologia e anatomia patologica, semeiotica e diagnostica - e descrisse anche i rapporti esistenti tra malattie orali e patologie sistemiche. Scrisse anche su argomenti più strettamente tecnici, di rado trattati nella letteratura scientifica italiana, quali l'occlusione, l'ortodonzia, l'odontoiatria conservativa, le lesioni cervicali $\left(\mathrm{I}_{3}\right)$ e la metallurgia applicata alla protesi dentaria. Nel "Ricettario odontoiatrico" (I4), pubblicato a Napoli nel I9I3, raccolse millesessantasette ricette, molte inedite, raggruppate per temi e precedute da nozioni di patologia e terapia delle patologie a cui erano destinate (9). Particolare attenzione rivolse all'igiene dentale e orale sostenendo la necessità del loro insegnamento fin dall'età infantile, sottolineando la necessità di procedere a regolari controlli negli scolari delle elementari e pubblicando, nel 1937 a Milano, un volume divulgativo intitolato "I denti e la salute" (15). Chiavaro ha lasciato anche un cospicuo testo di autobiografia scientifica: "Cinquant'anni di attività didattica e scientifica in Odontologia" ( 16 ), edito nel 1938 a Genova, del quale tratteremo più diffusamente in seguito.

\section{LA SCUOLA NAZIONALE DI ODONTOIATRIA E LE VICISSITUDINI AD ESSA COLLEGATE.}

Occupatosi inizialmente della riforma degli studi di Medicina e Chirurgia, Chiavaro dedicò poi molti suoi contributi a sostenere l'istituzione di una Scuola di Odontoiatria che avesse in comune i primi quattro anni con la Facoltà di Medicina e Chirurgia e gli ultimi due dedicati alla preparazione più specificatamente Odontoiatrica (9). Ma il successo ottenuto con la promulgazione del già citato Regio Decreto del 3r dicembre 1923 - che istituiva la Scuola Nazionale di Odontoiatria annessa all'Università di Roma e che avrebbe dovuto conferire la laurea in Odontoiatria, cui si aggiungevano altri tre decreti attuativi (relativi all'esame di Stato ed all'istituzione della professione) - fu di breve durata. Dieci mesi dopo, il Regio Decreto Legislativo del 
I6 ottobre $1924 \mathrm{n}^{\circ} 1755$ (fig.4), pubblicato sulla Gazzetta Ufficiale del Regno d'Italia il I3 novembre I924, abrogava i predetti decreti che non avevano ancora trovato applicazione, introducendo invece una norma che rendeva obbligatorio il corso di Clinica Odontoiatrica nella Facoltà medica e stabilendo la possibilità di una Specializzazione in Odontoiatria (non vincolante però per l'esercizio della professione) solo dopo aver conseguito la Laurea in Medicina e Chirurgia. La prima specializzazione in Odontoiatria fu istituita a Bologna, ad opera del prof.

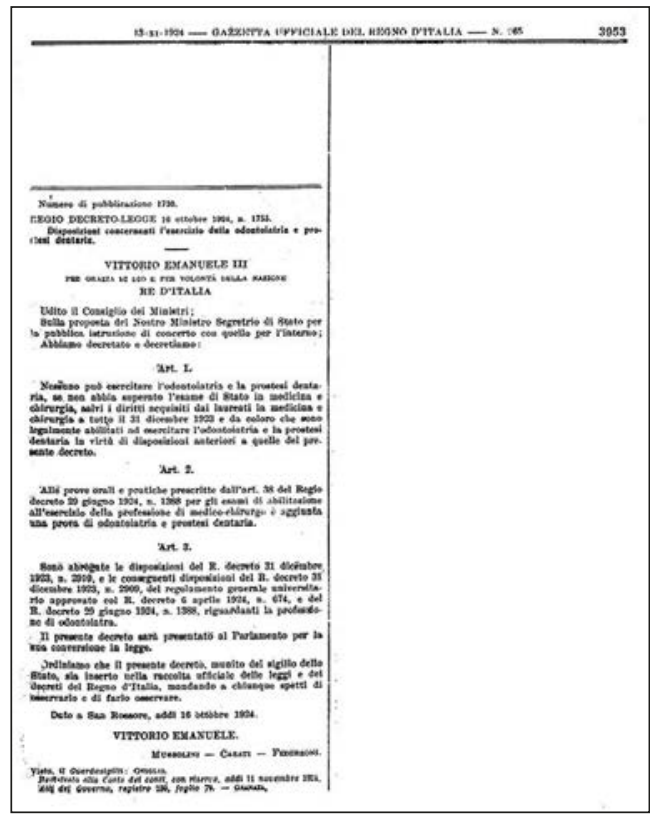

Fig. 4-Copia originale del Regio Decreto Legislativo I6 ottobre $1924 \mathrm{n}^{\circ} 1755$ - Regno d'Italia.

Arturo Beretta ( 17 ). Chiavaro, che aveva continuato a battersi strenuamente sulla stampa specializzata e non per mantenere i decreti o almeno attuarli in via sperimentale, evidentemente "irritò" le gerarchie fasciste e nel I928, senza preavviso alcuno, venne trasferito d'ufficio all'Università di Genova e sostituito alla cattedra romana dal prof. Amedeo Perna, prima Deputato e poi Senatore con "importanti agganci nel Partito Nazionale Fascista" (I8).

Rileggendo le opere di argomento non strettamente scientifico ancora reperibili di Chiavaro, balzano subito all'occhio tre aspetti fondamentali: l'amore incondizionato e costante per l'Odontoiatria in ogni suo aspetto, la ritrosìa a parlare delle sue vicende personali, le conseguenze psicologiche del "trasferimento" del 1928 che in pratica spezzò la sua attività libero-professionale e bloccò quella accademica. Interessante a tali riguardi ci sembra la possibilità di leggere le sue parole in tempi differenti della vita. Anzitutto riportiamo alcuni stralci del testo "Storia e Progressi dell'Odontoiatria" (I9), prolusione al corso universitario che Chiavaro, nel Igo6, tenne agli studenti di Medicina di Roma:

"l'Odontoiatria non è dunque solo scienza, ma è anche arte, ed in ciò differisce dagli altri rami della medicina e chirurgia. (Essa è un campo) ... a 
coltivare il quale non tutti possono riuscire, ma solo quelli che, oltre a possedere le conoscenze generali medico-chirurgiche, hanno una certa disposizione meccanica ed un ben sviluppato senso artistico. No, non bastano le sole conoscenze generali della medicina e chirurgia e le nozioni di patologia e cura delle malattie dei denti, per essere in grado di divenire esperto in Odontoiatria. Lo sviluppo sempre crescente di questa nuova ed importante scienza ed arte, ha fatto sì che nelle nazioni, ove essa ha maggiormente progredito, si è cominciato a suddividerla in diversi rami, professati da specialisti (...). Oramai è tempo che anche in Italia si contribuisca al progresso di questa Scienza, ed essa questo aspetta da voi. In voi non può mancare quell'intelligenza e quella genialità, che sono state sempre qualità invidiate al popolo italiano; non manchi, dunque, la buona volontà al lavoro assiduo. Il lavoro assiduo è lavoro proficuo: esso frutta a noi stessi il benessere morale e materiale; esso è il fattore principale della grandezza di un popolo. Solo col lavoro si acquista la coscienza di quello che noi siamo, di quello che noi possiamo. E siamo all'alba del giorno, in cui noi, popolo della più giovane tra le più grandi nazioni del mondo, potremo guardare serenamente, senza sentimento di sconforto, le civiltà degli altri popoli più evoluti, e possiamo ancora e dobbiamo conquistare il primato nelle scienze e nelle arti, per esserne dispensatori a tutto il mondo, come lo furono per lungo tempo i nostri grandi e gloriosi antenati".

Invece, l'unica nota personale "sfuggita" a Chiavaro, e che siamo riusciti a trovare, è nel lavoro che egli scrisse in risposta ad un altro articolo intitolato "Americanismo", comparso nella sua rivista "Annali di Odontologia", in cui il famoso direttore di un'altrettanto famosa Scuola Odontoiatrica nord americana parlava, come esempio di Americanismo, di un suo allievo che lavorava nei mesi estivi come facchino in una stazione ferroviaria per pagarsi gli studi odontoiatrici. Chiavaro scrive testualmente (fig.5) "Io parlo nel

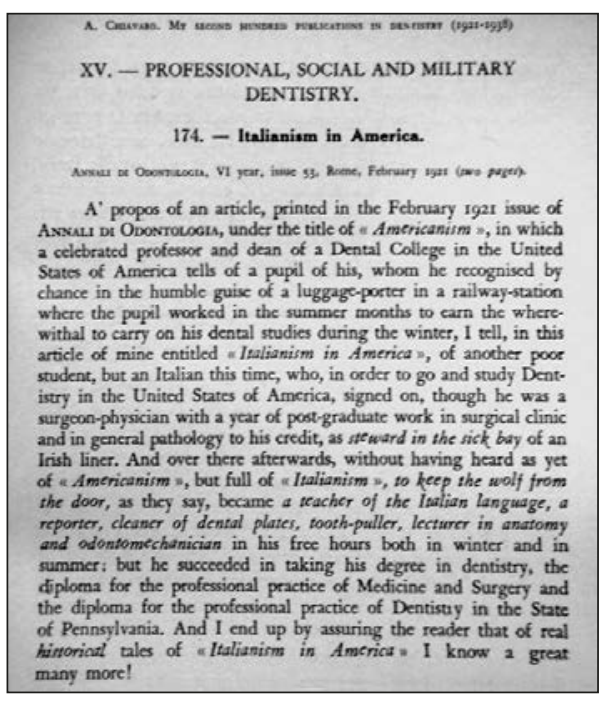

Fig. 5 - Pagina relativa all'articolo di A. Chiavaro "Italianismo in America". 
mio articolo, dal titolo "Italianismo in America" di un altro povero studente, italiano questa volta, che per recarsi a studiare Odontoiatria negli Stati Uniti d'America si fece assumere come infermiere su una nave di linea irlandese benché fosse Medico-Chirurgo con un anno di perfezionamento in Clinica Chirurgica e in Patologia generale. E una volta giunto là, senza aver provato ancora "Americanismo", ma pieno di "Italianismo", per sbarcare il lunario come si dice, lavorò come insegnante di italiano, giornalista, pulitore di apparecchi dentali, cavadenti, insegnante di anatomia e odontotecnico nelle ore libere dell'estate e dell'inverno" ... ed è evidente che, nonostante l'uso della terza persona, si riferisca a sé stesso. Il documento probabilmente più significativo della vicenda umana di Chiavaro è il già citato "Cinquant'anni di attività didattica e scientifica in Odontologia" (fig. 6) (r6) costituito da una prima parte, scritta nel I920, nella cui introduzione di oltre trenta pagine l'autore presenta con orgoglio ed entusiasmo la sua attività, i traguardi raggiunti e le speranze per l'Odontoiatria italiana in un prossimo futuro, ma anche da una seconda parte (scritta nel 1939) nella cui introduzione, in tutto una pagina, scrive mestamente "Ho pensato che fosse meglio non presentare qui una sintesi della mia carriera didattica e scientifica dal I92I al I938. Mi sembra però necessario puntualizzare che in questi 18 anni sono stato trasferito dall'Università di Roma a quella di Genova e che per circa un anno sono tornato negli Stati Uniti d'A-

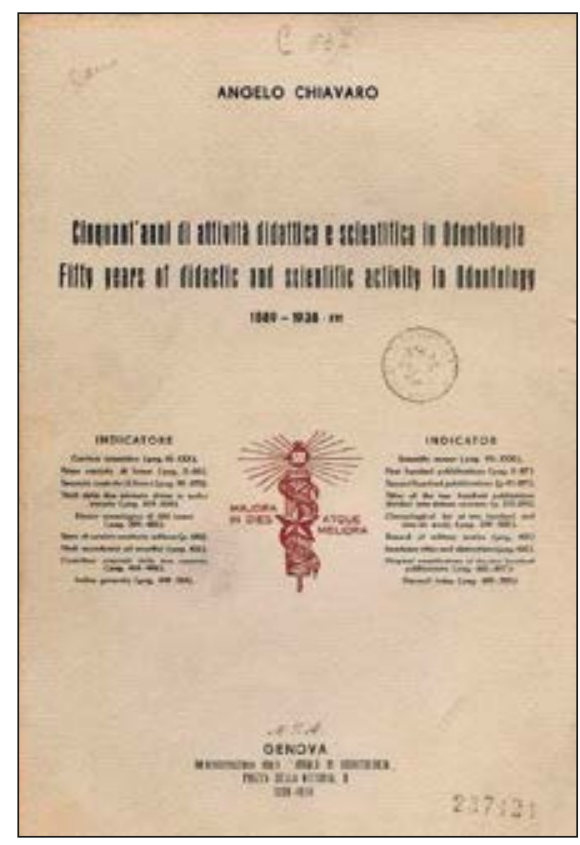

Fig. 6 - Pagina di copertina di "Cinquant'anni di attività didattica e scientifica in Odontologia". merica per insegnare e studiare" (I6): in particolare, nel I93I, tenne dei corsi al Dental College dell'Università di Berkeley, California (20) . E, in un altro punto di questa seconda parte, scrive - a proposito di una serie di lavori di embriologia, istologia, e anatomia comparate iniziati nel 1927 - "Avendo perduto un'enormità del mio tempo nell'organizzazione della riforma della legislazione dentale italiana, nell'istituzione della Laurea in Odontoiatria, 
nella fondazione della Scuola Nazionale in Roma, nelle conseguenze della promulgazione di queste leggi, che poi sono state abrogate senza essere applicate e nel trasferimento da me non richiesto dalla Cattedra di Odontoiatria di Roma, che avevo fondato nel 1915, a quella di Genova non ho più trovato il tempo e la tranquillità di spirito necessari per continuare.." (I6). Ricordiamo che Chiavaro scriveva queste ultime note nell'anno in cui l'Italia si legava militarmente alla Germania nazista, che l'avrebbe trascinata nell'orribile avventura della II Guerra Mondiale, e - in generale - in uno dei periodi più bui della storia italiana nel quale qualunque testo a stampa (ma anche di posta privata) veniva vagliato dalla censura fascista e poteva provocare gravi conseguenze. Nel periodo che va dal trasferimento a Genova sino alla morte (r944), Chiavaro continuò comunque la sua attività accademica con dedizione e serietà e trasformò, come abbiamo già ricordato, gli "Annali di Odontologia" in "Archivio Chiavaro", quasi a sottolineare sdegnosamente l'isolamento in cui l'avevano lasciato i Docenti suoi colleghi. Negli anni successivi alla fine della II Guerra Mondiale, mentre l'Odontoiatria compiva passi da gigante in tutto il mondo, in Italia le Scuole di Specializzazione post laurea in Medicina continuarono a formare pochi specialisti, quasi tutti di orientamento essenzialmente chirurgico. Così "nei primi anni '6o si contavano rz scuole di specializzazione" (2I) che poi aumentarono nei due decenni successivi sino a 22, ma dalle quali uscivano 250-350 specialisti l'anno, che non bastavano ai fabbisogni del Paese. Verso la fine degli anni '7o dello scorso secolo però un piccolo gruppo di Cattedratici, tra i quali vanno certamente segnalati i professori Luigi Capozzi e Antonio Baratieri (22), iniziò - anche su stimolo del Ministero dell'Università e Ricerca scientifica che chiedeva di ottemperare alle non più eludibili richieste dell'Unione Europea - a elaborare un progetto per l'istituzione del Corso di Laurea in Odontoiatria anche in Italia. Ciò avvenne il i6 aprile i980 quando, con la pubblicazione sulla Gazzetta Ufficiale della Repubblica Italiana $n^{\circ}$ 104, divenne esecutivo il Decreto del Presidente della Repubblica del 28 febbraio 1980, n. 315 riguardante "l'Istituzione del Corso di Laurea in Odontoiatria e protesi dentaria presso la Facoltà di Medicina e Chirurgia": Angelo Chiavaro aveva finalmente vinto ... ben trentasei anni dopo la sua morte. Infine, pochi mesi dopo l'uscita dei primi laureati in Odontoiatria, con la legge n. 409 del 24 luglio 1985, venne istituita la Professione Sanitaria di Odontoiatra: "Una nascita, quella del 1985, che non fu comunque facile, essendo stata determinata soprattutto dalla necessità di rispondere ad un obbligo della Comunità europea, poco sentito dal mondo accademico, e venendo osteggiata da una fetta di medici inclini 
ad esercitare la professione odontoiatrica in maniera corollaria a quella medica” (2I). Nel 1994 venne chiamato dall'Università di Perugia ad insegnare al corso di Laurea in Odontoiatria il primo Professore Associato laureato in Odontoiatria e, nel 2002, venne chiamato dall'Università di Roma "La Sapienza" il primo Professore Ordinario laureato in Odontoiatria. Il resto è cronaca di oggi.

\section{Conclusioni}

Angelo Chiavaro, al quale dovrebbero andare costantemente il pensiero reverente e la gratitudine di ogni laureato italiano in Odontoiatria, fu un grande ed incompreso precursore, che tentò di inserire un'impostazione moderna dell'Odontoiatria in un Paese come l'Italia dei primi del ı9oo, impreparata a ricevere tale visione, già vincente in tutti gli altri Paesi più avanzati. Egli ha lasciato, comunque, un vasto retaggio di conoscenze (è stato tra i primi in Italia a trattare di Ortodonzia, Gnatologia e Parodontologia) ma soprattutto l'esempio di un impegno civile, nato dal suo amore per l'Odontoiatria, che scontò duramente con un immeritato ostracismo sopportato con virile pazienza. 


\section{Bibliografia}

1. McCauley HB- The first dental college: emergence of dentistry as an autonomous profession - J Hist Dent. 51(1): 41-5, 2003

2. Schulein TMA - Chronology of dental education in the United States. J Hist Dent. 52(3):97-108, 2004

3. Morgenstern H - La mise en place de l'Ecole dentaire de Paris et de l'Ecole dentaire de France: organisation, administration, programme des études. Les lois du 12 juillet 1875 et du 18 mars 1880 et la fondation des écoles dentaires. Actes. Société française d'histoire de l'art dentaire 10: 32-39, 1995

4. Blankenstein F - 110 Jahre Zahnärztliches Institut Berlin, 1884-1994: Festschrift. Quintessenz Verlags-GmbH, Berlin, 1994

5. Doubleday FN. The history of the Dental School of Guy's Hospital. Guys Hosp Gaz. 62 (1562):152-5, 1948

6. Zampetti P. - Principio stomatologico o autonomia odontoiatrica? Storia di un percorso complesso- http://www.ildentistamoderno.com/principio-stomatologico-o-autonomia-odontoiatrica-storia-di-un-percorso-complesso/

7. Zampetti P - Gli uomini che fecero l'Odontoiatria italiana: Angelo Chiavaro (1870-1944 -) Doctor Os, 22 (6), 2011

8. Zampetti P., Barbon G. - Cento anni di Odontoiatria in Italia : 1912-2012-Actes. Société française d'histoire de l'art dentaire, 18: 90-92, 2013

9. Cantani A. - Chiavaro Angelo - Voce del Dizionario Biografico degli Italiani Volume 24,1980

10. Necrologio di Angelo Chiavaro - Rassegna trimestrale di Odontoiatria., XXVI (1945), pp. 39-40

11. Eramo S. Fioratti R., Genovesi C. , Gallottini L. "Not Just Two Million Teeth: Giovanni Battista Orsenigo, Monk Dentist” J Hist Dent 58(3): 141-146, 2010

12. Chiavaro A. - Semeiotica e diagnostica speciale odontoiatrica - in Trattato di semeiologia fisica e diagnostica chirurgica generale e speciale, diretto da D. TaddeiTorino, UTET, 1924

13. Eramo S, Baldi M, Marci MC, Monaco A - Histopathological and therapeutical aspects of cervical lesions - Minerva stomatologica 52 (1-2), 69-74, 2002

14. Chiavaro A. - Ricettario odontoiatrico - Napoli, Soc. Tip. Napoletana, 1913

15. Chiavaro A. - I denti e la salute - Milano, Mondadori, 1937

16. Chiavaro A. - Cinquant'anni di attività didattica e scientifica in Odontologia; Fifty years of didactic and scientific activity in odontology: 1889-1938. Genova, Buona Stampa, 1939

17. De Carolis C, Bravi M, Pagano S, Eramo S - Arturo Beretta e la "teoria trofomicrobica della carie” - Acta Medico-Historica Adriatica 11 (2): 339-348, 2013 
18. Vicarelli A, Spina E - Professionalization and Professionalism: The Case of Italian Dentistry Professions and Professionalism 5 (3 ): $1-18$ http://dx.doi. org/10.7577/pp.1324

19. Chiavaro A -Storia e Progressi dell'Odontoiatria - Il Policlinico-Sez. Chirurgica - XIII: 215 -255 1906

20. University of California Bulletin - College of Dentistry - Announcements 1930-1931 - Part XII, pag. 49 - University of California Press, Berkeley, 1930

21. Melato M, Rizzardi C - Le radici della moderna professione odontoiatrica in Italia ed il contesto europeo in Medicina \& Storia - Saggi, Firenze University Press, Firenze - VIII, (16): 119-145, 2008

22. Baratieri A.- La 2a riunione di esperti sull'insegnamento universitario dell'odontoiatria indetta dal Comitato dell'insegnamento superiore e della ricerca del Consiglio d'Europa in Strasburgo il 21-22 marzo 1974- Rivista italiana di stomatologia, 29 (1974), n. 7-9

\section{RiAssunto}

La nascita della Laurea in Odontoiatria in Italia è stata molto contrastata e solo nel I980 ha visto la sua effettiva realizzazione. Molto "istruttiva" al riguardo è la vicenda relativa all'istituzione nel 1924, periodo della presa di potere da parte del Fascismo, di una "Scuola Nazionale di Odontoiatria" presso l'Università di Roma, revocata appena dieci mesi dopo. Il maggiore sostenitore e propugnatore della Scuola, il prof. Angelo Chiavaro, venne dopo qualche anno "punito" con il trasferimento d'ufficio dall'Università di Roma a quella di Genova. Presentiamo alcune brevi note relative alla biografia di questo coraggioso precursore e alla vicenda di cui fu protagonista.

Parole chiave: Chiavaro; odontoiatria; laurea odontoiatria. 